\title{
A REMARK ON STRONG PSEUDOCONVEXITY FOR ELLIPTIC OPERATORS
}

\author{
BY VICTOR GUILLEMIN ${ }^{1}$
}

Communicated by Shlomo Sternberg, October 19, 1971

The purpose of this note is to give a kind of intrinsic characterization of second order elliptic operators.

Let $p(x, D)$ be an $m$ th order elliptic operator defined on an open subset $U$ of $\boldsymbol{R}^{n}$. Let $p_{m}(x, \xi)$ be its leading symbol. Let $\varphi$ be a smooth function on $U$ with the property that $\operatorname{grad} \varphi \neq 0$ when $\varphi=0$. The hypersurface, $\varphi=0$, is said to be strongly pseudoconvex at a point $x \in \varphi^{-1}(0)$ if

$$
\sum \frac{\partial^{2} \varphi}{\partial x_{j} \partial x_{k}} p_{m}^{(j)}(x, \xi) \overline{p_{m}^{(k)}}(x, \xi)+\tau^{-1} \operatorname{Im} \sum p_{m, k}(x, \xi) \overline{p^{(k)}(x, \xi)}>0,
$$

for all $\xi=\eta+i \tau \operatorname{grad} \varphi$, where $\eta \in \boldsymbol{R}^{n}$ and $0 \neq \tau \in \boldsymbol{R}$, satisfying the equations :

$$
p_{m}(x, \xi)=0=\sum p_{m}^{(j)}(x, \xi) \partial \psi / \partial x_{j} .
$$

(See Hörmander [2, Chapter 8].)

If $p$ is a second order operator and its leading symbol is real, then, for $\eta, N \in \boldsymbol{R}^{n}$ not multiples of each other, the equations

$$
p_{2}(x, \eta+\tau N)=0=\sum p_{2}^{(j)}(x, \eta+\tau N) N_{j}
$$

have no solutions, so condition (1) is satisfied trivially. This proves

Proposition 1. If $p(x, D)$ is second order and its leading symbol is real, then every hypersurface is strongly pseudoconvex.

In this note we will prove a result in the other direction, namely,

PROPOSITION 2. If $n \geqq 3$ and every hypersurface is strongly pseudoconvex then $p(x, D)$ is second order.

REMARK 1 . If there exist vectors $\eta, N$ satisfying (3) it is easy to construct a $\varphi$, with $\operatorname{grad} \varphi(x)=N$, violating (1). Therefore for every surface with normal, $N$, at $x$ to be strongly pseudoconvex at $x$ it is necessary and sufficient that there be no $\eta, N$ satisfying (3). Hence Proposition 2 can be reformulated as a simple algebraic assertion, namely,

Proposition 3. Let $p(\zeta), \zeta \in C^{n}$, be a homogeneous polynomial of degree $m$. Assume $n \geqq 3$, and assume $p$ satisfies the following conditions:

AMS 1970 subject classifications. Primary 35-XX.

${ }^{1}$ Supported by NSF GP-22927.

Copyright (c) American Mathematical Society 1972 
(a) $p$ has no real zeros except 0 .

(b) For any fixed pair of vectors $\xi$ and $\eta$ in $\boldsymbol{R}^{n}$, $\xi$ and $\eta$ not multiples of each other, the equation $p(\xi+\tau \eta)=0, \tau \in C$, has simple roots.

Then $m=2$.

REMARK 2. It is easy to see that condition (4) is equivalent to the following condition:

$$
\sum \frac{\partial p(\xi)}{\partial \xi^{i}} \xi^{i} \neq 0 \text { when } p(\xi)=0, \xi \in C^{n} \neq 0 .
$$

Remark 3. It is enough to prove Proposition 3 when $n=3$. In fact choose coordinates so that the coefficient of $\xi_{1}^{m}$ in $p(\xi)$ is nonzero. If the theorem is true in dimension 3 then $p\left(\xi_{1}, \xi_{2}, \xi_{3}, 0, \ldots, 0\right)$ is of degree 2 so $m=2$.

We will assume from now on that $n=3$ and that $p(\xi)$ satisfies condition (5). Since $p(\xi)$ is homogeneous in $\xi$ its zero variety defines an algebraic curve, $\gamma$, in $P^{2}(C)$. By $(5)$, $\operatorname{grad} p(\xi) \neq 0$ when $p=0$ so this curve is nonsingular.

We identify $P^{2}(C)$ with the set of one-dimensional subspaces of $C^{3}$. Given $l \in P^{2}(C)$, let $L_{l}=\left\{\xi \in C^{3}, \xi \in l\right\}$ and let $L$ be the vector bundle on $P^{2}(C)$ whose fiber at $L$ is $L_{l}$. Let $L_{\gamma}=L 1 \gamma$. Given $l \in P^{2}(C)$, let $\xi$ be a nonzero vector on $l$ and let

$$
H_{l}=\left\{\left(a_{1}, a_{2}, a_{3}\right) \in C^{3}, \sum \frac{\partial p}{\partial \xi^{i}}(\xi) a_{i}=0\right\} .
$$

Let $H$ be the vector bundle on $\gamma$ whose fiber at $l$ is $H_{l}$. By Euler's identity $L_{\gamma} \subset H$. Let $J=H / L_{\gamma}$.

LEMMA 1. The Chern class $c_{1}(J)=0$.

Proof. Let $E$ be the trivial bundle over $\gamma$ with fiber $C^{3}$. Let $\bar{L}_{\gamma}$ be the line bundle over $\gamma$ whose fiber at $l \in P^{2}(C)$ is $\{\bar{\xi}, \xi \in l\}$. It is easy to see that $\bar{L}$ is the dual bundle of $L$ so $c_{1}(\bar{L})=-c_{1}(L)$. The composite bundle map $L \rightarrow E \rightarrow E / H$ is bijective by condition $(5)$ so $c_{1}(E / H)=c_{1}(\bar{L})$. Since $E$ is trivial, $0=c_{1}(E)=c_{1}(L)+c_{1}(J)+c_{1}(E / H)=c_{1}(L)+c_{1}(J)-c_{1}(L)$ $=c_{1}(J)$. Q.E.D.

Let $T$ be the holomorphic tangent bundle of $\gamma$. There is a canonical identification $T \cong \operatorname{Hom}\left(L_{\gamma}, J\right)$, so $c_{1}(T)=c_{1}(J)-c_{1}\left(L_{\gamma}\right)=-c_{1}\left(L_{\gamma}\right)$. By Riemann-Roch, $c_{1}(T)=2-2 g, g$ being the genus of $\gamma$ (see Gunning [1, p. 110]) so $c_{1}\left(L_{\gamma}\right)=2 g-2$. On the other hand $c_{1}\left(L_{\gamma}\right)=-m$.

(ProOf. Let $\omega$ be a linear functional on $C^{3}$. Then $\omega$ defines a section of $L^{*}$ whose zero set is the hyperplane $\omega=0$. The Chern class of $L^{*} 1 \gamma$ is the number of points in which this hyperplane intersects $\gamma$, which is just the 
degree of $p$ for hyperplanes in general position.)

To summarize we have proved the equality

(6)

$$
2 g-2=-m \text {. }
$$

Since $m$ is positive this can be satisfied only for $g=0$ and $m=2$. Q.E.D.

\section{BiblogRAPHY}

1. R. C. Gunning, Lectures on Riemann surfaces, Princeton Math. Notes, Princeton Univ. Press, Princeton, N.J., 1966. MR 34 \# 7789.

2. L. Hörmander, Linear partial differential operators, Die Grundlehren der math. Wissenschaften, Band 116, Academic Press, New York; Springer-Verlag, Berlin, 1963. MR 28 \#4221.

Department of Mathematics, Massachusetts Institute of Technology, Cambridge, MassachusetTS 02139 